\title{
An improved microscale method for extraction of phenolic acids from maize
}

\author{
Mariana Zavala-López and Silverio García-Lara* (i)
}

\begin{abstract}
Background: Phenolic acids are a major group of secondary metabolites widely distributed in plants. In the case of maize, the major proportion of these metabolites occurs in the edible grain and their antioxidant activities are associated with improvements in human health. However, conventional extraction of secondary metabolites is very time consuming and generates a substantial amount of solvent waste. One approach to resolve these limitations is the use of microscale approaches, which minimize the quantity of solvents required, as well as the sample amounts and processing times. The objective of this work was to develop an improved microscale method for extraction of phenolic acids from maize and to compare it with a conventional extraction method.

Results: The improved microscale extraction method, coupled with an HPLC-DAD detection method, allowed identification of ferulic acid, $p$-coumaric acid in its free and bound form, and some diferulic acids. In its free form, p-coumaric acid ranged in content from 2.4 to $6.5 \mathrm{\mu g} / \mathrm{g}$ dry weight ( $\mathrm{dw}$ ) using the conventional method and 7.7 to $54.8 \mu \mathrm{g} / \mathrm{g}$ dw using the improved microscale method. Free ferulic acid content ranged from 2.6 to $12.9 \mu \mathrm{g} / \mathrm{g} \mathrm{dw}$ for the conventional method and 16.8 to $181.7 \mu \mathrm{g} / \mathrm{g} \mathrm{dw}$ for the improved microscale method. In its bound form, p-coumaric acid ranged in content from 6.0 to $30.6 \mu \mathrm{g} / \mathrm{g} \mathrm{dw}$ for the traditional method and 34.4 to $138.6 \mathrm{\mu g} / \mathrm{g} \mathrm{dw}$ for the improved microscale method. Bound ferulic acid ranged from 131.8 to $427.5 \mu \mathrm{g} / \mathrm{g} \mathrm{dw}$ for the conventional method and 673.8 to $1702.7 \mu \mathrm{g} / \mathrm{g}$ dw for the improved microscale method. The coefficient of variation associated was lower for the improved microscale method than for the conventional method, thereby assuring the replicability of the process.

Conclusions: The improved microscale method proposed here increases the extraction power and batch capacity, while reducing the sample quantity, solvent amounts and extraction time. It also achieves a better replicability with a lower coefficient of variation than is possible with conventional extraction.
\end{abstract}

Keywords: Extraction, Phenolic acid, Maize, Microscale

\section{Background}

Phenolic compounds are a major group of secondary metabolites widely distributed in plants. The phenolics found in maize have drawn attention because of their associated benefits for human health. These health effects are mainly associated with the antioxidant activity of these compounds that aids in protection of the plant itself against pests [1]. The phenolic profile varies according to the matrix analyzed: in the case of maize, the major

\footnotetext{
*Correspondence: sgarcialara@itesm.mx

Biotechnology Center, School of Engineering and Science, Tecnologico

de Monterrey, Campus Monterrey, Eugenio Garza Sada 2501,

C.P. 64849 Monterrey, N.L., Mexico
}

proportion of phenolics are bound to the cell wall, and the two most prominent phenolic compounds are the phenolic acids, trans-ferulic acid and $p$-coumaric acid [2].

Analysis of these metabolites requires an efficient extraction procedure that provides a high extraction rate as well as a high specificity for the compounds of interest. Conventional extraction procedures involve the use of different solvents that take into consideration the polarity of the compounds to be extracted [3]. A typical procedure can be divided into two major steps: free phenolic extraction and bound phenolic extraction. The first step consists of an alcohol extraction to obtain the soluble 
compounds, while the second step is an alkali hydrolysis to free the bound phenolics from the cell wall; this is followed by a series of several washes to purify the sample [4]. One of the major drawbacks of this procedure is its requirement for large quantities of different solvents (i.e., methanol, hexane and ethyl acetate), which generates a significant quantity of toxic solvent waste [3]. The procedure is also very time consuming, making the handling of several samples at once a challenging task.

Alternative methods for phenolic acid extraction have been developed, mainly using techniques of microwaveassisted extraction [5], pulsed-electric field [6], ultrasound, and supercritical fluid extraction [3]. However, these techniques still present other major disadvantages, such as requirements for further purification processes [7], lack of identification power [8] and lower recovery yield [9], or they are still under development for their use in maize. For these reasons, microscale approaches have gained attention in recent years, as they have the potential to resolve the major issues of conventional extractions by minimizing the quantity of solvents and sample required while shortening the processing time. These adaptations allow the handling of larger sets of samples per batch in a shorter amount of time [10-12]. The objective of the present work was to develop an improved microscale extraction method for phenolic acids in maize and to compare its efficacy to that of a conventional macroscale extraction method.

\section{Methods}

\section{Materials}

A group of nineteen temperate yellow hybrids maize materials was used for the evaluation of the conventional and microscale phenolic acid extraction procedures. All maize hybrids were harvested during 2014 and kindly donated by a Local private company. The ears were dried, shelled, milled, and stored at $4{ }^{\circ} \mathrm{C}$ until further processing. A list of materials, along with proximal analysis, can be found in Additional file 1. Maize kernels were ground to a fine powder $(<1 \mathrm{~mm})$ using two grinding steps (Krups GX4100, MX, followed by Retsch MM400) and passed through a sieve (US. 60). The ground maize material was stored at $-20^{\circ} \mathrm{C}$.

\section{Reagents}

$\mathrm{NaOH}$ and Folin-Ciocalteu reagents were purchased from Macron, CH. $2 \mathrm{M} \mathrm{HCl}$ was obtained from J.T. Baker, MX. N-hexane was purchased at DEQ, MX. Ethyl acetate was obtained from Burdick \& Jackson, Muskegon, MI. All HPLC solvents and alcohols were obtained from BDH, West Chester, PA. HPLC grade (> 99\%) standards for gallic acid, trifluoroacetic acid, p-coumaric acid and ferulic acid were purchased from Sigma-Aldrich, St. Louis, MO.

\section{Conventional phenolic acid extraction}

The conventional phenolic acid extraction methodology used was a modified method of Adom \& Liu [2]. Briefly, $10 \mathrm{~mL}$ of $80 \%$ ethanol were added to $1 \mathrm{~g}$ of ground maize sample. The samples were incubated at $25^{\circ} \mathrm{C}$ for $15 \mathrm{~min}$ with constant agitation at $500 \mathrm{rpm}$ (Vortemp 1550, Labnet, Woodbridge, NJ) and then centrifuged (VWR 1814, USA) for $10 \mathrm{~min}$ at $10,000 \mathrm{rpm}$ and $4{ }^{\circ} \mathrm{C}$. The supernatant was decanted and stored at $-20{ }^{\circ} \mathrm{C}$ until analysis. The residue pellet was used for extraction of bound phenolics, as follows. Ten $\mathrm{mL}$ of $2 \mathrm{M} \mathrm{NaOH}$ were added to the residue pellet. The resuspended pellet was mixed at $3000 \mathrm{rpm}$ on a vortex mixer (Vortex VWR, USA). An alkaline hydrolysis treatment was carried out by incubation at $90{ }^{\circ} \mathrm{C}$ for $1.5 \mathrm{~h}$ and constant agitation at $500 \mathrm{rpm}$. The samples were then acidified with $14 \mathrm{~mL}$ of $2 \mathrm{M} \mathrm{HCl}$ and the $\mathrm{pH}$ of the samples was verified as being between $\mathrm{pH} 2$ and 3 using $\mathrm{pH}$ strips (Macherey-Nagel, DK). Lipids were removed by adding $12 \mathrm{~mL}$ of $\mathrm{n}$-hexane to the samples, followed by vortexing for $5 \mathrm{~min}$ at $2500 \mathrm{rpm}$, incubation at $25{ }^{\circ} \mathrm{C}$ for $10 \mathrm{~min}$ with constant agitation at $500 \mathrm{rpm}$, and centrifugation for $10 \mathrm{~min}$ at $10,000 \mathrm{rpm}$. The upper hexane phase in the resulting three-layered system was discarded and this procedure was repeated twice more, for a total of three $\mathrm{n}$-hexane washes. Ten $\mathrm{mL}$ of ethyl acetate were then added to recover the phenolic acids. The samples were mixed at $3000 \mathrm{rpm}$, incubated at $25{ }^{\circ} \mathrm{C}$ for $10 \mathrm{~min}$ with constant agitation at $500 \mathrm{rpm}$ and then centrifuged again for $10 \mathrm{~min}$ at $10,000 \mathrm{rpm}$. The upper ethyl acetate phase was recovered from the resulting three-layer system that formed. The ethyl acetate was removed by evaporating to dryness in an extraction hood and the sample was resuspended in $5 \mathrm{~mL}$ of $50 \%$ methanol. This procedure was repeated to complete five ethyl acetate washes. The extracts were stored at $-20{ }^{\circ} \mathrm{C}$ until analysis.

\section{Improved microscale phenolic acid extraction}

The improvement in phenolic acid extraction, achieved through the use of a microscale process, was performed as follows. Instead of ethanol, $80 \%$ methanol was used as the extraction solvent for soluble phenolic acids; $0.7 \mathrm{~mL}$ were added to $50 \mathrm{mg}$ of maize sample and mixed for $5 \mathrm{~min}$ at $2500 \mathrm{rpm}$ on a vortex (Vortex VWR, USA). The sample was incubated at $25{ }^{\circ} \mathrm{C}$ for $15 \mathrm{~min}$ at a constant agitation of $500 \mathrm{rpm}$ (Vortemp 1550, Labnet, Woodbridge, NJ), followed by centrifugation (VWR 1814, USA) for $10 \mathrm{~min}$ at $5000 \mathrm{rpm}$. The supernatant was decanted and stored at $-20{ }^{\circ} \mathrm{C}$ until analysis. The bound phenolics were extracted from the pellet residue formed in the previous step using a microscale procedure. First, the volume of $2 \mathrm{M} \mathrm{NaOH}$ added to the pellet was reduced from 10 to $0.5 \mathrm{~mL}$. The samples were flushed with nitrogen gas 
to protect them from oxygen degradation and vortexed for $5 \mathrm{~min}$ at $2500 \mathrm{rpm}$.

The alkaline hydrolysis was conducted at a temperature maintained at $90{ }^{\circ} \mathrm{C}$ and a constant agitation at $500 \mathrm{rpm}$, but the procedure time was reduced to $1 \mathrm{~h}$ (compared to $1.5 \mathrm{~h}$ ). After hydrolysis, the sample was acidified with $0.5 \mathrm{~mL}$ of $2 \mathrm{M} \mathrm{HCl}$, and a value of $\mathrm{pH} 2$ was verified with $\mathrm{pH}$ strips; otherwise the $\mathrm{pH}$ was adjusted with the appropriate amount of $2 \mathrm{M} \mathrm{HCl}$. Lipid removal was achieved by adding only $0.8 \mathrm{~mL}$ of $\mathrm{n}$-hexane to the samples, which were then vortexed for $5 \mathrm{~min}$ at $2500 \mathrm{rpm}$, incubated at $25{ }^{\circ} \mathrm{C}$ for $10 \mathrm{~min}$ with a constant agitation of $500 \mathrm{rpm}$, and centrifuged for $10 \mathrm{~min}$ at $5000 \mathrm{rpm}$. The hexane layer from the resulting three-layered system was discarded (upper phase). This procedure was repeated in order to complete two n-hexane washes. The bound phenolic acids were recovered by adding $0.8 \mathrm{~mL}$ of ethyl acetate, mixing the sample for $5 \mathrm{~min}$ at $2500 \mathrm{rpm}$, incubating at $25^{\circ} \mathrm{C}$ for $10 \mathrm{~min}$ with a constant agitation of $500 \mathrm{rpm}$ and centrifuged for $10 \mathrm{~min}$ at $5000 \mathrm{rpm}$. The ethyl acetate layer (upper phase) from the three-layered system formed was recovered. The number of repetitions of this process was reduced to only three complete ethyl acetate washes. Ethyl acetate was evaporated to dryness in an extraction hood and the sample was resuspended in $200 \mu \mathrm{L}$ of $50 \%$ methanol, filtered through a $0.45 \mu \mathrm{m}$ GHP and Nylon filter (Pall Life Sciences, Ann Harbor, MI), and stored at $-20{ }^{\circ} \mathrm{C}$ until further analysis.

\section{Determination of total free and bound phenolic acids}

The total free and bound phenolics extracted using the improved and conventional extraction methods were quantified using the Folin-Ciocalteu assay according to Urias-Peraldí et al. [13]. Briefly, $100 \mu \mathrm{L}$ of $10 \%$ folin reagent were added to $20 \mu \mathrm{L}$ of sample in a 96-well microplate. After $5 \mathrm{~min}$, the reaction was neutralized with 80 $\mu \mathrm{L}$ of $\mathrm{Na}_{2} \mathrm{CO}_{3}(7.5 \% \mathrm{w} / \mathrm{v})$. Incubation was performed for $2 \mathrm{~h}$ at room temperature. Total phenolic acids were quantified using a microplate reader (Synergy ${ }^{\mathrm{TM}}$ HT MultiDetection, BioTek, Inc., Winooski, VT) at $765 \mathrm{~nm}$. Gallic acid was used as a standard and total phenolic content was expressed as $\mu \mathrm{g}$ of gallic acid equivalents per $100 \mathrm{mg}$ dry weight (GAE/100 mg dw).

\section{Determination of free and bound phenolic acids by HPLC}

Extracted phenolic acids were analyzed according to the method of Ayala-Soto et al. [14] using an HPLC (Agilent 1100 Santa Clara, CA) coupled with a photodiode array (PDA) detector (Agilent G1315D, Santa Clara, CA). Linear gradient elution was performed with HPLC-grade water (acidified to $\mathrm{pH} 2$ with trifluoroacetic acid) and acetonitrile, at a flow rate of $0.6 \mathrm{~mL} / \mathrm{min}$ at $25{ }^{\circ} \mathrm{C}$. Phenolic acids were separated on a Zorbax SB-Aq, $4.6 \mathrm{~mm}$
ID $\times 150 \mathrm{~mm}(3.5 \mu \mathrm{m})$ reverse phase column. The Chemstation software (for LC; Copyright $\odot$ Agilent Technologies, 1990-2003) was used to process the data and command the equipment. Peak identification of trans-ferulic acid and $p$-coumaric acid was based on the retention times of known standards. Diferulic acids were identified according to the retention times reported previously [14-16] and were reported as equivalents of ferulic acid.

\section{Statistical analysis}

Phenolic acid extractions were done in triplicate and total randomization of replicates was used to minimize the bias of the assay. Statistix ${ }^{\circledR}$ v. 8 was used for the statistical analysis of the data. Statistical analysis included analysis of variance (ANOVA, $\alpha=0.05 \%$ ) between methods of extraction and between samples. Significant differences between the means of samples were further analyzed by least significant difference (LSD, $\alpha=0.05$ ). Box plot diagrams were used to show the dispersion within each method of extraction for the different maize samples and to compare between methods, as well as to reveal the dispersion in the coefficient of variation associated to each extraction method. A p value $<0.05$ was considered statistically significant.

\section{Results}

The new microscale method for phenolic acid extraction was validated in tandem with the conventional method by extracting 19 maize samples by both the conventional and the improved methods. Table 1 summarizes the principal differences between both methodologies, considering the amount of sample, procedure time, required solvents and generated solvent waste. The required sample was reduced from 1000 to $50 \mathrm{mg}$ with the microscale method; this represents a reduction of $95 \%$ of the required sample. Drying time was decreased over $70 \%$, solvent consumption for the bound phenolic extraction was lowered by $65 \%$ and solvent waste was reduced by in $67 \%$. The microscale protocol also increased the number of samples that an operator was able to process per batch. Sixty-six samples were handled in a single batch, compared with the 9 samples handled by the conventional method, representing an increase of $633 \%$. Comparison of the conventional method with the microscale method proposed here revealed several advantages of the improved method, especially when sample quantity was a constraint. The improved method allowed for a reduction in the quantity of sample and solvents required, extraction time, and solvent waste generated, while increasing the number of samples analyzed per batch.

Total phenolic acids were quantified in free and bound forms by the conventional and improved methods. 
Table 1 Comparison of the main steps of the process by conventional and improved methods

\begin{tabular}{lccr}
\hline Step & $\begin{array}{l}\text { Conventional } \\
\text { method }\end{array}$ & $\begin{array}{l}\text { Improved } \\
\text { method }\end{array}$ & $\boldsymbol{\Delta}^{*}$ (\%) \\
\hline Required sample (mg) & 1000 & 50 & 95 \\
Extraction time & & 30 & -100 \\
$\quad$ Free phenolics (min) & 15 & 10 & 17 \\
$\quad$ Bound phenolics (h) & 12 & 2 & 71 \\
Drying time (days) & 7 & 46 & \\
Consumed solvents & & 376 & 65 \\
Free phenolics $(\mathrm{mL})$ & 90 & 106 & 67 \\
$\quad$ Bound phenolics $(\mathrm{mL})$ & 1080 & 66 & -633 \\
Solvents waste $(\mathrm{mL})$ & 324 & 9 &
\end{tabular}

$\Delta^{*}$ : Difference between conventional and improved methods expressed as percentages

a According to the capabilities of the available centrifuge equipment
Figure 1 shows a similar rate of extraction for total phenolic acids in free form by both extraction methodologies. In the free form, phenolic acids extracted by the conventional method ranged from 166.4 to $605.3 \mathrm{mg}$ $\mathrm{GAE} / 100 \mathrm{~g} \mathrm{dw}$ while the microscale method achieved a similar extraction range that fluctuated from 125.2 to $682.0 \mathrm{mg} \mathrm{GAE} / 100 \mathrm{~g} \mathrm{dw}$; this difference was not statistically significant (Additional file 2). Comparison of the coefficient of variations for the two methodologies showed that the improved protocol had a reduced range, where most of the data fell below 0.05 , whereas the conventional method had a higher range, where most of the data rested between 0.12 and 0.03 .

Both methodologies were significantly different in terms of the phenolic concentration determined in the bound form (Fig. 1). Bound phenolic acids extracted by the conventional method ranged from 248.3 to $1158.4 \mathrm{mg}$ $\mathrm{GAE} / 100 \mathrm{~g} \mathrm{dw}$, whereas the improved method achieved
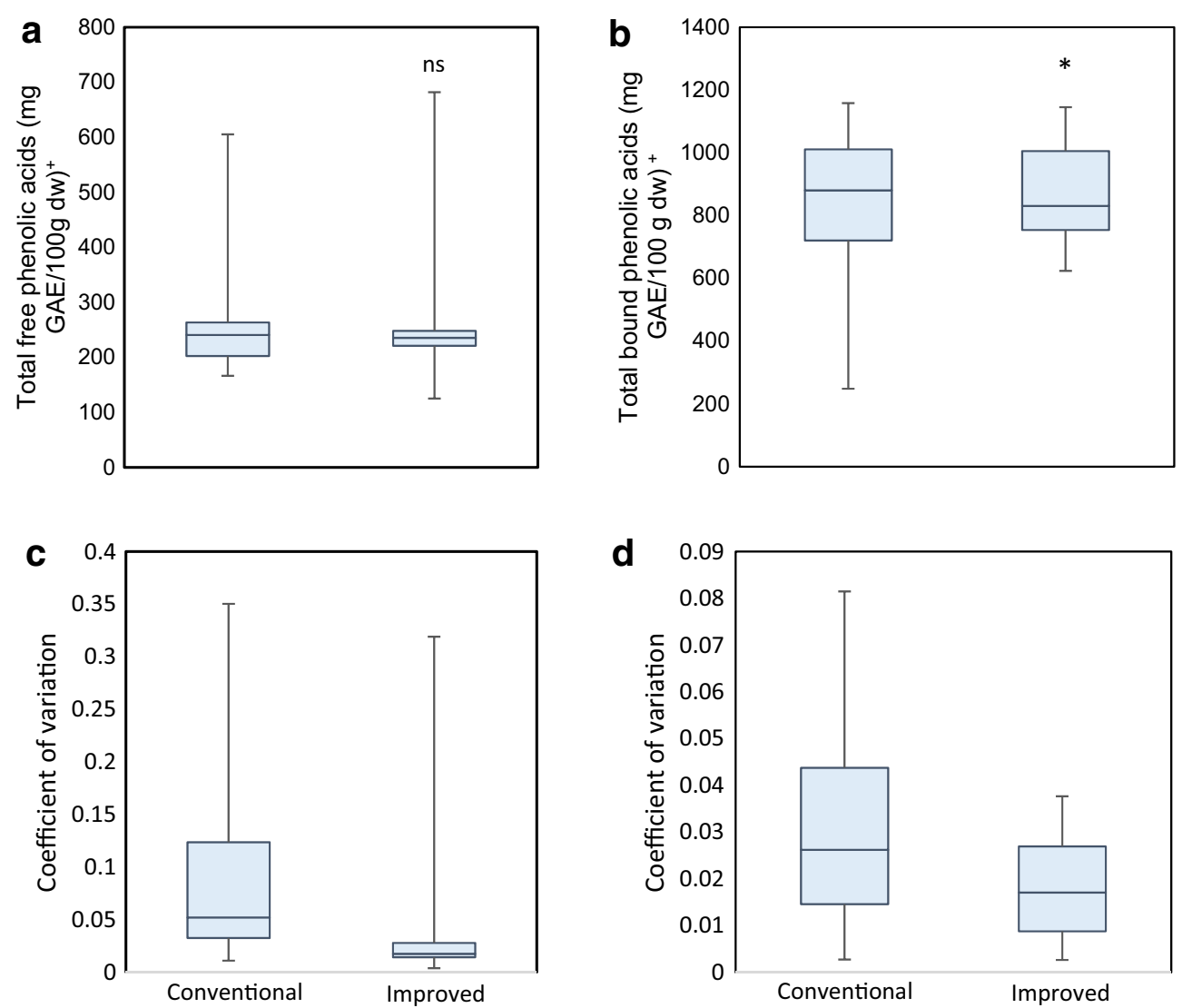

Fig. 1 Box plot comparison of total phenolic acids in maize seeds. a Total free phenolic acids. b Total bound phenolic acids. c Coefficient of variation of total free phenolic acid quantification; $\mathbf{d}$ coefficient of variation of total bound phenolic acids. Horizontal line represents the median of total values. Vertical line represents the maximum and minimum value. Box covers the 75 and 25 percentiles. $\left[^{+}\right.$Results are expressed as mg of gallic acid equivalent per $100 \mathrm{~g}$ of dry weight (mg GAE/100 g dw). Significant differences between the methods were established by ANOVA ( $a=0.05)$, ns no significance, $\left.{ }^{*} p<0.05 ;{ }^{* *} p<0.001 ;{ }^{* * *} p<0.001\right]$ 
a higher extraction that ranged from 623.9 to $1165.3 \mathrm{mg}$ GAE/100 g dw (Additional file 3). The lowest coefficient of variation for both methodologies was under 0.01; however, the highest value with the conventional method reached 0.08 , whereas the improved method did not exceed 0.04 . The improved method achieved an extraction of total phenolic acids that was as efficient as the conventional method, and superior to the conventional method for bound phenolic acids, with a lower variation in the amounts of both total free and bound phenolic acids.

The results for free phenolic acids, quantified individually by HPLC, are shown in Fig. 2. Significant differences were found between the extraction methods for free $p$-coumaric and trans-ferulic acid. In the free form, the microscale method ranged from 7.7 to $54.8 \mu \mathrm{g} / \mathrm{g} \mathrm{dw}$ for $p$-coumaric acid and 16.8 to $181.7 \mu \mathrm{g} / \mathrm{g} \mathrm{dw}$ for transferulic acid, respectively (Additional file 3). Thus, the improved method increased the extraction by over sevenfold for $p$-coumaric acid and eightfold for trans-ferulic acid. The improved method showed an important lower variation when compared with the conventional method, as the coefficient of variation was lower than 0.15 when compared with the coefficient of variation of over 0.3 observed with the conventional method. The conventional method also showed a higher range of variation from nearly 0 to over 0.8 . Thus, the improved microscale method achieved a higher extraction for both $p$-coumaric and trans-ferulic acids in their free form, with a reduced variation, when compared to the conventional method.

Individual bound phenolic acids extracted by the conventional and improved methods and quantified by HPLC are shown in Fig. 3. Significant differences were found between the extraction methods for both the bound phenolic acids quantified. The microscale method achieved a greater extraction of $p$-coumaric acid, ranging from 34.4 to $138.6 \mu \mathrm{g} / \mathrm{g} \mathrm{dw}$, compared to the range of 6.0 to $30.6 \mu \mathrm{g} / \mathrm{g} \mathrm{dw}$ obtained using the conventional method. Extraction of trans-ferulic acid was also increased with the improved microscale method, ranging from 673.8 to $1702.7 \mu \mathrm{g} / \mathrm{g} \mathrm{dw}$, compared to 131.8 to $427.5 \mu \mathrm{g} / \mathrm{g} \mathrm{dw}$ for the conventional method. The improved method also
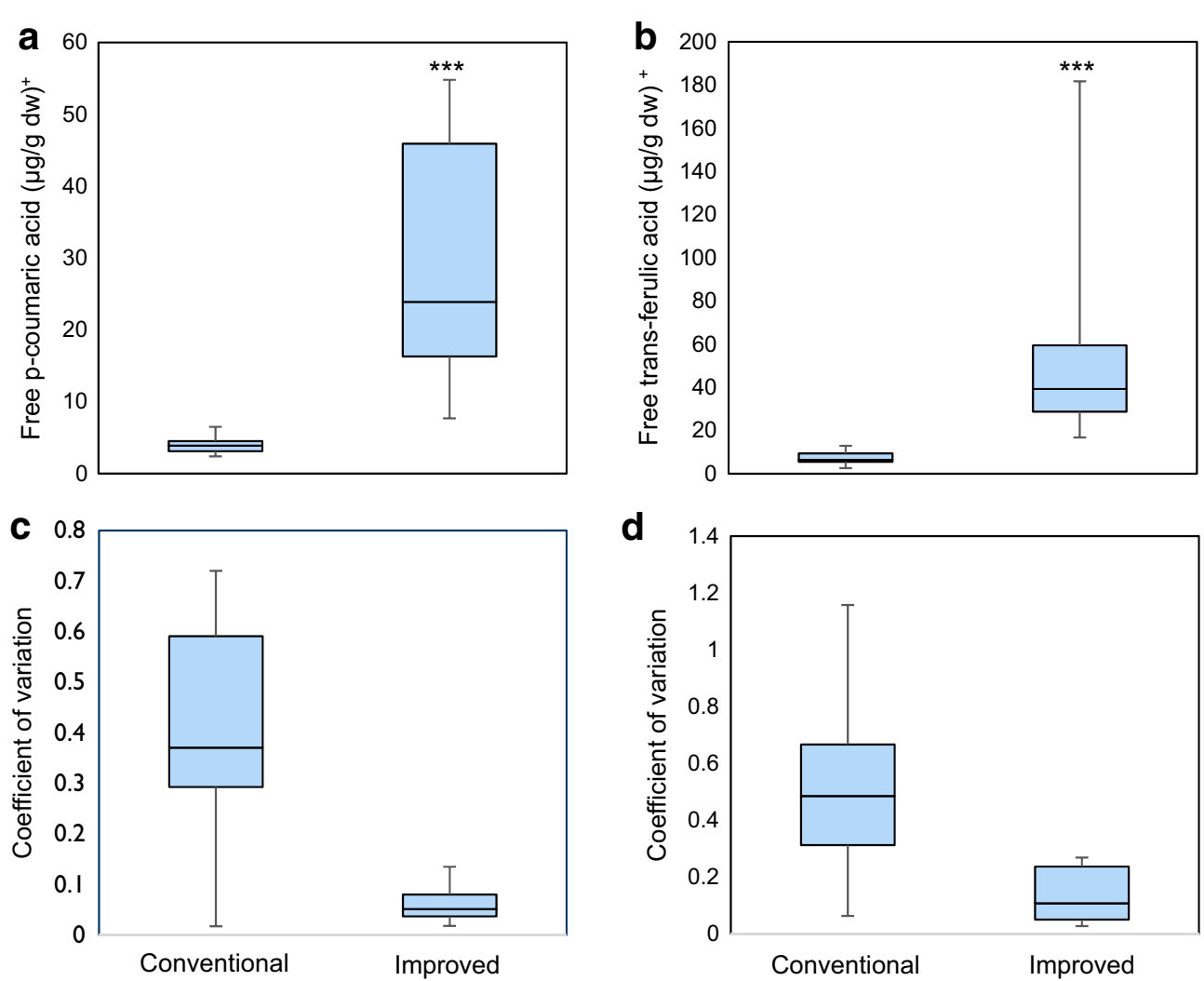

Fig. 2 Box plot comparison of free phenolic acids extracted from maize seeds by the conventional and improved methods. a Determination of $p$-coumaric acid. b Determination of trans-ferulic acid. c Standard deviation for $p$-coumaric acid. d Standard deviation for ferulic acid. Horizontal line represents the median. Vertical line represents the maximum and minimum value. Box covers the 75 th and 25 th percentiles. $\left[{ }^{+}\right.$Results are expressed as $\mu \mathrm{g}$ per $\mathrm{g}$ dry weight $(\mu \mathrm{g} / \mathrm{g} \mathrm{dw}$ ). Significant differences between the methods were established by ANOVA $(a=0.05)$, ns no significance, $\left.{ }^{*} p<0.05 ;{ }^{* *} p<0.001 ;{ }^{* * *} p<0.001\right]$ 

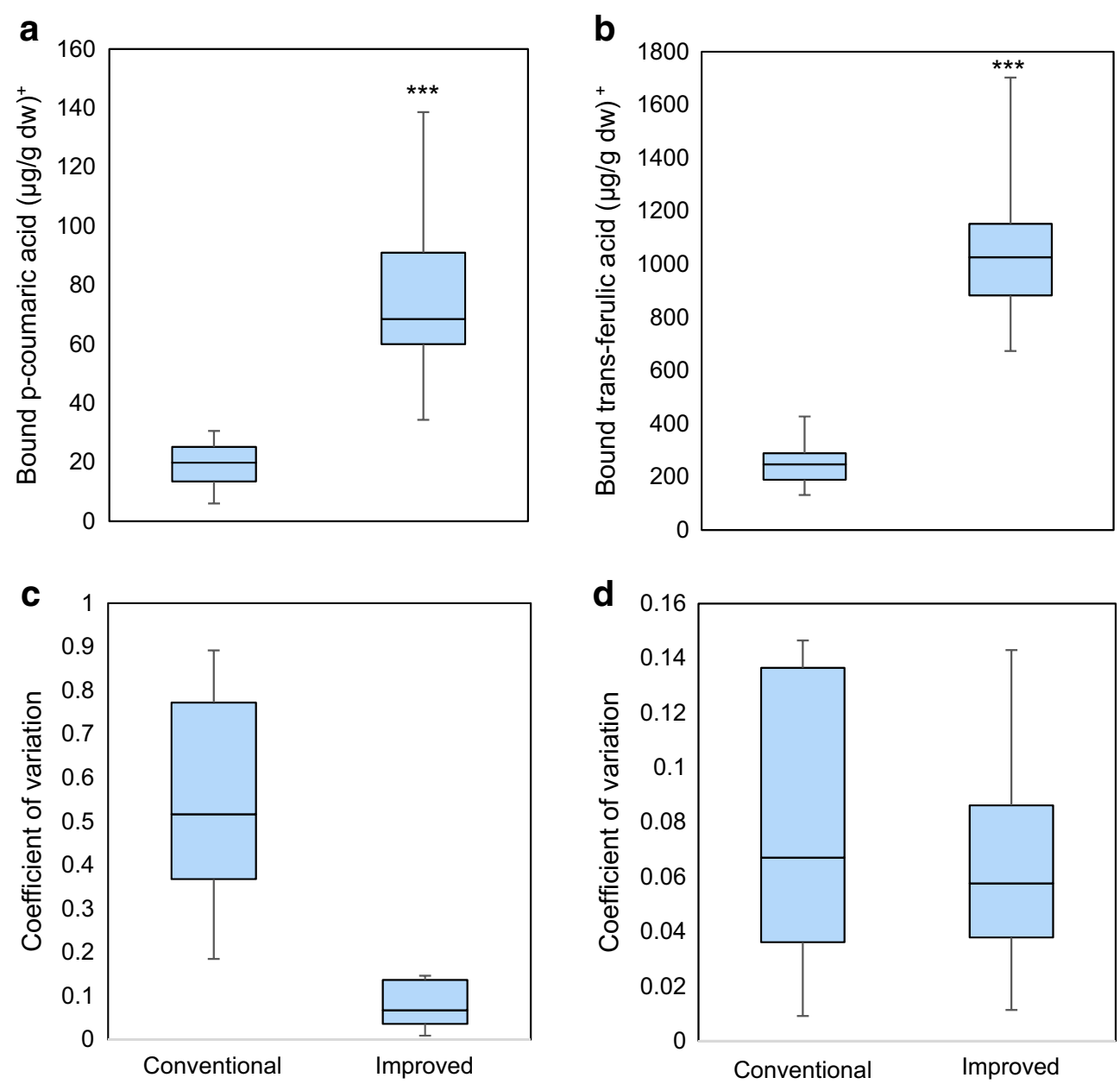

Fig. 3 Box plot comparison of bound phenolic acids in maize seeds extracted by the conventional and improved methods. a Determination of $p$-coumaric acid. b Determination of trans-ferulic acid. c Standard deviation of $p$-coumaric acid. $\mathbf{d}$ Standard deviation ferulic acid. Horizontal line represents the median. Vertical line represents the maximum and minimum value. Box covers the 75 th and 25 th percentiles. [ ${ }^{+}$Results expressed as $\mu \mathrm{g}$ per $\mathrm{g}$ dry weight $(\mu \mathrm{g} / \mathrm{g} \mathrm{dw})$. Significant differences between the methods were established by ANOVA $(a=0.05)$, ns: no significance, ${ }^{*} p<0.05$; $\left.{ }^{* *} p<0.001 ;{ }^{* *} p<0.001\right]$

showed a reduced coefficient of variation for $p$-coumaric acid (lower than 0.15) while that for trans-ferulic acid showed a similar range of variance. However, most of the quantifications of trans-ferulic acid lay between 0.04 and 0.08 , while those for the conventional method centered between 0.04 and 0.14 . Therefore, the improved method achieved a higher extraction for both $p$-coumaric and trans-ferulic acid in their bound forms, with a reduced variation, when compared to the conventional method.

Isomers of diferulic acids were also quantified using both extraction methods, as shown in Table 2. Both extraction methods allowed the detection and quantification of 5-5 diferulic acid (5-5 DFA) and diferuloylputrescine (DFP). Significant differences were found between both extraction methods for the extraction of diferulic acids. The conventional method gave amounts of 8.6 and $6.6 \mu \mathrm{g}$ FAE/g dw for 5-5 DFA and DFP, respectively, whereas the improved method gave values of 22.5 and 13.4 $\mu \mathrm{g} \mathrm{FAE} / \mathrm{g} \mathrm{dw}$ for 5-5 DFA and DFP, respectively.

Figure 4 shows a comparison of the chromatograms obtained for the conventional and the improved methods. In addition to 5-5 DFA and DFP, the improved method allowed the identification and quantification of the diferulic isomers 8-5 diferulic acid, 8-0-4 diferulic acid and 5-5 DFA benzofurane. Two aromatic amides, p-coumaroyldiferuloyl putrescine and diferuloylputrescine, were also identified in some samples with a higher precision than with the conventional method. Thus, the improved microscale method achieved a better resolution and definition of diferulic acid peaks in the chromatogram, allowing their proper identification and quantification. 
Table 2 Comparison of conventional macroscale and improved microscale methods for extracted bound phenolic acids founded in whole maize hybrids kernels

\begin{tabular}{|c|c|c|c|c|c|c|c|c|}
\hline & \multicolumn{2}{|c|}{$p$-Coumaric acid ${ }^{a}(\mu \mathrm{g} / g \mathrm{dw})$} & \multicolumn{2}{|c|}{ trans-Ferulic acid ${ }^{a}(\mu \mathrm{g} / \mathrm{g} \mathrm{dw})$} & \multicolumn{2}{|c|}{ 5-5 DFA ${ }^{b}(\mu \mathrm{g} \mathrm{FAE} / \mathrm{g} \mathrm{dw})$} & \multicolumn{2}{|c|}{$\mathrm{DFP}^{\mathrm{b}}(\mu \mathrm{g} F \mathrm{FA} / \mathrm{g} \mathrm{dw})$} \\
\hline & Conv & Improve & Conv & Improve & Conv & Improve & Conv & Improve \\
\hline Minimum & 6.0 & 34.4 & 131.8 & 673.8 & 8.6 & 22.5 & 6.6 & 13.4 \\
\hline Mean & 19.4 & 74.2 & 249.7 & 1029.9 & 12.9 & 64.9 & 20.2 & 25.0 \\
\hline Maximum & 30.6 & 138.6 & 427.5 & 1702.7 & 17.3 & 131.5 & 31.5 & 49.9 \\
\hline LSD & 18.6 & 10.9 & 270.1 & 121.9 & 8.7 & 8.2 & 18.2 & 19.9 \\
\hline C.V. & 58.9 & 8.9 & 53.8 & 7.2 & 40.5 & 7.6 & 54.7 & 48.1 \\
\hline ANOVA & $* * *$ & & $* * *$ & & $* * *$ & & * & \\
\hline
\end{tabular}

Conv conventional method. Improve improved method. DFA diferulic acid, DFP diferuloylputrescine. Significant differences between the methods were established by ANOVA $(a=0.05)$, NS no significance, ${ }^{*} p<0.05 ;{ }^{* *} p<0.001 ;{ }^{* *} p<0.001$. LSD least significant difference $(a=0.05)$

a Results are expressed as the average of three replicates as $\mu \mathrm{g}$ per $\mathrm{g}$ dry weight $(\mu \mathrm{g} / \mathrm{g} \mathrm{dw}$ )

b Results are expressed as the average of three replicates as $\mu \mathrm{g} \mathrm{FAE/g} \mathrm{dw:} \mu \mathrm{g}$ of ferulic acid equivalents per $\mathrm{g} \mathrm{dw}$

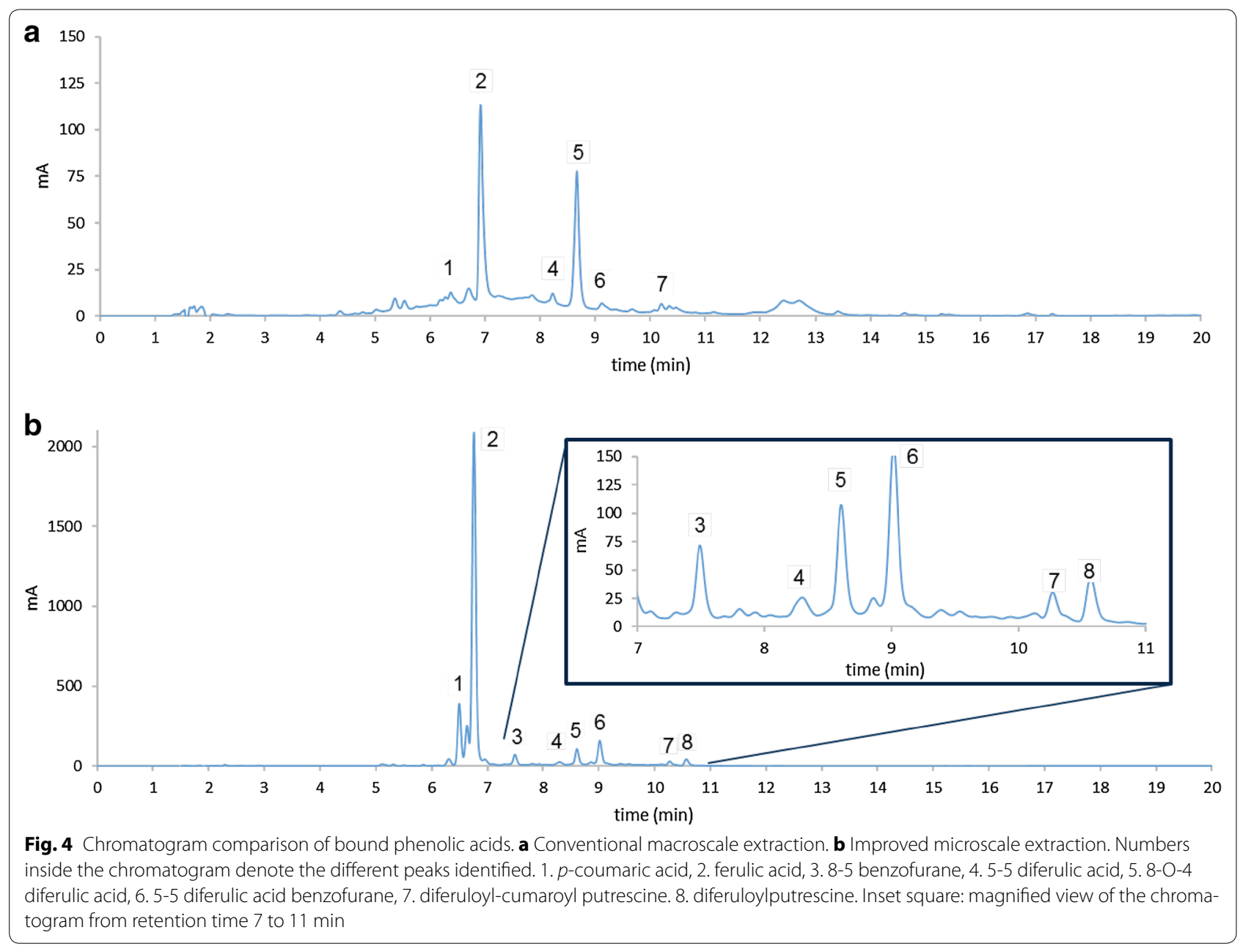

\section{Discussion}

Conventional extraction of phenolic acids involves the use of different solvents that take advantage of their polarities to make a solid-liquid extraction. The major drawbacks of conventional procedures are the use of large quantities of different solvents, which generate a significant volume of toxic waste [3], and the highly time-consuming nature of the method, which makes 
the handling of several samples at once a challenging task.

In the present study, we compared a conventional extraction method with an improved, microscale method in yellow maize samples, and found several advantages of the improved method. It allowed a reduction in the required quantity of sample and solvents, in the extraction time, and in the amount of solvent waste generated, while increasing the number of samples that could be handled per batch. The enhanced performance of the improved method was achieved through important modifications of the conventional method, which involve solvents and solvent volumes for extraction, sample:solvent ratios, and the duration of the processes in the method. Different solvents, like alcohols, acetone, diethyl ether and ethyl acetate, have been used for phenolic acid extraction [3]. The use of only organic solvents is restricted, as extremely polar phenolic acids would not be effectively extracted. Aqueous solutions of methanol or ethanol (70-80\% alcohol) are the usual solvents of choice for the first step of phenolic acid extraction; however, although methanol is more efficient at extraction, its use is limited because ethanol is considered a safer and more environmentally friendly solvent [17]. Consequently, the low solvent volume required for the improved method could make methanol an environmentally acceptable choice for extraction.

The contents of total free and bound phenolic acids, as evaluated by the conventional and improved extraction methods, gave results that were in accordance with previous reports that quantified total free and bound phenolic acids in maize [13, 18-21]. Similar amounts of total free phenolic acids were extracted using either methodology, but a greater amount of total bound phenolics was extracted with the improved method. This represents a unique and novel advantage, particularly in light of the drastic reduction in the amount of sample required. Modifications to the method for scaling down did not negatively affect the extraction of total phenolic acids. Both methodologies confirmed that the bound form represented over $75 \%$ of the total phenolic acids, in agreement with the results reported previously by Adom and Liu [2].

The phenolic acids bound to cell walls have typically been extracted using a number of different treatments. For example, alkaline or acidic hydrolysis are both possible choices. Alkaline hydrolysis breaks the ester bonds linking phenolic acids to the cell wall, while acid hydrolysis breaks glycosidic bonds and solubilizes sugars, leaving ester bonds intact [3]. The alkaline hydrolyses of cereals usually use $\mathrm{NaOH}$, most commonly at a concentration of $2 \mathrm{M}$ [22]. Comparisons of alkaline and acidic hydrolyses have indicated that different phenolic acid extraction profiles can be favored in each case. Alkaline hydrolysis achieves a higher extraction of total phenolic acids [23], and particularly of trans-ferulic acid and p-coumaric acid, which can suffer degradation under acidic conditions at high temperatures [24, 25].

Higher extractions with longer durations of hydrolysis have been reported [26]. However, prolonged times of alkaline hydrolysis can decrease the phenolic acid content [27]. This effect of varying hydrolysis times reveals the equilibrium required for phenolic acid extraction: longer extraction times increase the chance of higher extraction rates but also of oxidation [17]. In the case of trans-ferulic acid, higher hydrolysis times have been reported to decrease the recovery to $63 \%$ [2]. Therefore, finding an optimal duration for hydrolysis is important to allow higher extraction of phenolic acids without promoting degradation of these compounds. In the conventional methodology, alkaline hydrolysis is performed for $1.5 \mathrm{~h}$, whereas for the improved methodology, this time was reduced to $1 \mathrm{~h}$. In both methods, the temperature was set at $90^{\circ} \mathrm{C}$ and the extracts were flushed with nitrogen gas to prevent oxidation. $\mathrm{NaOH}$ was added at $2 \mathrm{M}$ in both methodologies and the solvent:sample ratio was maintained at 10:1. The improved method may establish a better equilibrium for the hydrolysis process by the adjustment of the hydrolysis duration, thereby achieving a better extraction of bound phenolic acids by a combination of increased extraction and less degradation.

The adjustment of $\mathrm{pH}$ after the hydrolysis treatment with the improved method had a major influence on the phenolic acid content. A precise adjustment to $\mathrm{pH} 2$ must be performed to allow proper quantification of bound phenolic acids, when compared to the broader range (pH 2-3) used in the conventional method. The effect of $\mathrm{pH}$ on phenolic compound stability has been evaluated previously and is related to the structure of each phenol. Both $p$-coumaric acid and trans-ferulic acid have been identified as having a high stability to $\mathrm{pH}$ changes, although more complex phenolic compounds, including diferulic acids, have been found to be more susceptible to $\mathrm{pH}$. The stability to $\mathrm{pH}$ has been associated with the number of $\mathrm{OH}$ groups present, their position and the number of substituents in the benzene ring [28].

A phenolic acid extraction method must consider the solvent:sample ratio as well as the number of replicate extractions, as both will affect the recovery performance [29]. In the conventional extraction, a total of 5 ethyl acetate washes are used, with a solvent:sample of 10:1. By contrast, the improved method uses only 3 ethyl acetate washes with a solvent:sample of 16:1. The increased solvent:sample ratio in the improved method could explain the higher extraction rate, as more solvent was available to interact with the matrix without having 
a saturation effect. The increase in phenolic acid extraction in response to an increasing solvent:sample ratio has been reported previously, although a compromise was needed to achieve a high phenolic acid extraction without an excessive increase in the solvent required, considering the high solvent costs and solvent waste generation [30]. Clearly, the conventional method has areas of opportunity, especially in terms of the solvent:sample ratio, even when a higher number of washes is employed. As already mentioned, the conventional method was less efficient at extracting the bound phenolic acids when compared to the microscale method.

One interesting result was revealed by the comparison of quantifications following the two extraction methods. No significant difference was noted between the total free phenolic acids, but differences appeared for the individual free phenolic acids (trans-ferulic acid and $p$-coumaric acid). By contrast, total bound phenolic acid contents, as well as individual bound phenolic acid content, were significantly higher for the improved method than for the conventional method. Therefore, in the case of free phenolic acids, the quantification of total phenolic acids apparently fails to distinguish the improvement revealed by the HPLC quantification. The Folin-Ciocalteu (F-C) assay is generally the method of choice for quantification of total phenolic acids in food samples. This colorimetric assay is based on the rapid oxidation reaction of phenols in an alkali (sodium bicarbonate). The phenolates reduce the $\mathrm{F}-\mathrm{C}$ reagent, changing it into a blue pigment that is measured spectrophotometrically [31]. The $\mathrm{F}-\mathrm{C}$ reaction is used extensively, but several disadvantages have been documented, most of them regarding its lack of specificity. It is an assay based on reduction, so it does not distinguish between reducing compounds other than phenolic acids that could be present in the sample. The two most recognized compounds of concern are ascorbic acid (or dehydroascorbic acid) and reducing sugars (glucose and fructose) [32]. Recently, an assay involving the use of 80 different standards showed that a broad variety of compounds, including phenols, proteins, thiols, vitamins and amino acids, show reactivity with the $\mathrm{F}-\mathrm{C}$ reagent [33].

A lack of correlation has been reported previously between the $\mathrm{F}-\mathrm{C}$ assay and phenolic compounds quantified by HPLC. Sánchez-Rangel et al. [32] reported quantification of the 5 major phenolic compounds in strawberry, but they found no correlation between the phenolic content quantified by HPLC and by the $\mathrm{F}-\mathrm{C}$ assay, whereas a significant correlation was found between interfering substances and the $\mathrm{F}-\mathrm{C}$ assay. A significant effect of interfering compounds has also been found in other matrixes, like carrot and kiwi [32]. Andjelkovic et al. [34] found a moderate correlation between HPLC and F-C assays for phenolic extracts of olive oils. In the case of maize, these effects have not been reviewed, possibly because of the low content of reducing sugars and ascorbic acid (around 0.02\% [35] and $323 \mathrm{nmol} / \mathrm{g}$ fresh weight [36], respectively) and its higher content of phenolic compounds [2]. However, consideration of the presence of interferents might be important in the case of analysis of free phenolic acids. The free phenolic compounds in this study represented around 25\% of the total phenolic acids, which agrees with the values reported by García-Lara and Bergvinson [18]. Note also that the extraction of free phenolic compounds is mostly based on the polarity of the compounds relative to the polarity of aqueous methanol (70\%). The co-extraction of other polar molecules, like sugars, proteins and hydrophilic vitamins, during the extraction of free phenolic compounds is possible and could have significant effects on the $\mathrm{F}-\mathrm{C}$ assay.

Complex phenolic acids were also identified and quantified in their bound forms. The results from both methods were similar in terms of the identity of the extracted compounds, but the higher extraction by the improved method allowed for a better identification and quantification of these compounds. Complex phenolic acids, like isomers of diferulic acid, have been studied before and are mostly associated with resistance to biotic agents in maize varieties [1,24, 37, 38]. Phenolic acid amides, including diferuloylputrescine, have been associated with maize weevil resistance [39] and as antibiosis factors to large corn borer [40]. The importance of a correct profile generation for complex phenolic acids has been highlighted previously, as these compounds could aid in the identification of better varieties for maize improvement [1].

Another major effect of the improved method is a consistent reduction in the coefficient of variation, when compared to the conventional method. This reduction was found for the total phenolic acids as well as the individual compounds. The coefficient of variation is directly related to the standard deviation of the replicates within a sample quantification; therefore, a lower value represents a higher replicability of the extraction procedure [41]. Replicability is an essential quality for any laboratory assay, especially when a large number of samples is handled. The improved method proposed here assures high sample handling without compromising the replicability of the assay.

\section{Conclusions}

Conventional phenolic acid extraction takes advantage of differences in polarities to achieve a reasonable extraction rate. However, the high consumption of solvents and time make this extraction non-sustainable and a lowcapacity processing method. The improved, microscale 
method proposed here provides an approach to overcome these issues, gaining extraction power and batch capacity with lower sample quantity, solvents and time, while also achieving a better replicability with a lower coefficient of variation than the conventional extraction. Additionally, the improved microscale method represents an efficient alternative for the management of several samples at once for phenolic extractions from maize without compromising the replicability of the assay.

\section{Additional files}

Additional file 1. Determination of proximal analysis for maize varieties. * Results are expressed as the average of three replicates \pm standard deviation.

Additional file 2. Comparison of total phenolic acids determination by conventional and improved methods. ${ }^{*}$ Results are expressed as the average of three replicates as $\mathrm{mg}$ of gallic acid equivalent per $100 \mathrm{~g}$ dry weight (mg GAE/100 g dw). Min: minimum value of the data, Max: maximum value of the data. LSD: Least significant difference $(a=0.05)$.

Additional file 3. Comparison of free phenolic acids determination by conventional and improved methods. *Results are expressed as the average of three replicates as $\mu \mathrm{g}$ per $\mathrm{g}$ dry weight $(\mu \mathrm{g} / \mathrm{g} \mathrm{dw}$ ). Min: minimum value of the data, Max: maximum value of the data. LSD: Least significant difference $(a=0.05)$

\section{Authors' contributions}

MZ-L performed experimental work, acquired results, carried out the data analysis and interpretation, and wrote the manuscript. SG-L helped in writing the manuscript, designing the experiment, interpreting results and supervising the study. Both authors read and approved the final manuscript.

\section{Acknowledgements}

The authors are thankful to A. Duran-Kishi, D. Ochoa-Martinez and S. Ortiz-Islas for their excellent technical assistance during the development of this work. We also thank V. Rocha-Villarreal and E. Lopez-Tavera for the English review and S. Serna-Saldivar for the technical review.

\section{Competing interests}

The authors declare that they have no competing interests.

\section{Availability of data and materials}

The supporting material described in "Methods" section was included in Additional file 1. The supporting materials described in the "Results" section are included in Additional files 2 and 3.

\section{Consent for publication}

Not applicable.

\section{Ethics approval and consent to participate}

Not applicable.

\section{Funding}

This work was supported by Congreso Nacional de Ciencia y Tecnologia (Conacyt, Mexico funding: 388368), Monsanto (St. Louis, MO) and Tecnologico de Monterrey (Mexico).

\section{Publisher's Note}

Springer Nature remains neutral with regard to jurisdictional claims in published maps and institutional affiliations.

Received: 18 October 2016 Accepted: 3 October 2017

Published online: 10 October 2017
References

1. García-Lara S, Bergvinson DJ, Burt AJ, Ramputh Al, Díaz-Pontones DM Arnason JT. The role of pericarp cell wall components in maize weevil resistance. Crop Sci. 2004;44:1546-52.

2. Adom KK, Liu RH. Antioxidant activity of grains. J Agric Food Chem. 2002;50:6182-7.

3. Acosta-Estrada BA, Gutiérrez-Uribe JA, Serna-Saldívar SO. Bound phenolics in foods, a review. Food Chem. 2014;152:46-55.

4. Gutiérrez-Uribe JA, Rojas-García C, García-Lara S, Serna-Saldivar SO. Phytochemical analysis of wastewater (nejayote) obtained after lime-cooking of different types of maize kernels processed into masa for tortillas. J Cereal Sci. 2010;52:410-6.

5. Zhang $\mathrm{H}-\mathrm{F}$, Yang $\mathrm{X}-\mathrm{H}$, Wang $\mathrm{Y}$. Microwave assisted extraction of secondary metabolites from plants: current status and future directions. Trends Food Sci Technol. 2011;22:672-88.

6. Barba FJ, Parniakov O, Pereira SA, Wiktor A, Grimi N, Boussetta N, et al. Current applications and new opportunities for the use of pulsed electric fields in food science and industry. Food Res Int. 2015;77:773-98.

7. Wang L, Weller CL. Recent advances in extraction of nutraceuticals from plants. Trends Food Sci Technol. 2006;17:300-12

8. Chiremba C, Rooney LW, Beta T. Microwave-assisted extraction of bound phenolic acids in bran and flour fractions from sorghum and maize cultivars varying in hardness. J Agric Food Chem. 2012;60:4735-42.

9. Wu J-J, Lin J-C, Wang C-H, Jong T-T, Yang H-L, Hsu S-L, et al. Extraction of antioxidative compounds from wine lees using supercritical fluids and associated anti-tyrosinase activity. J Supercrit Fluids. 2009;50:33-41.

10. Kokosa JM. Advances in solvent-microextraction techniques. TrAC Trends Anal Chem. 2013;43:2-13.

11. Spietelun A, Marcinkowski Ł, De La Guardia M, Namieśnik J. Green aspects, developments and perspectives of liquid phase microextraction techniques. Talanta. 2014;119:34-45.

12. Butts-Wilmsmeyer $\mathrm{CJ}$, Bohn MO. High-throughput extraction of insoluble-bound ferulic acid in maize. Crop Sci. 2016;56(6):1-8.

13. Urias-Peraldí M, Gutiérrez-Uribe JA, Preciado-Ortiz RE, Cruz-Morales AS, Serna-Saldívar SO, García-Lara S. Nutraceutical profiles of improved blue maize (Zea mays) hybrids for subtropical regions. Field Crop Res. 2013;141:69-76.

14. Ayala-Soto FE, Serna-Saldívar SO, García-Lara S, Pérez-Carrillo E. Hydroxycinnamic acids, sugar composition and antioxidant capacity of arabinoxylans extracted from different maize fiber sources. Food Hydrocoll. 2014;35:471-5.

15. Dobbeerstein D, Bunzel M. Separation and detection of cell wall-bound ferulic acid dehydrodimers and dehydrotrimers in cereals and other plant materials by reversed phase high-performance liquid chromatography with ultraviolet detection. J Agric Food Chem. 2010;58:8927-35.

16. Waldron KW, Parr AJ, Ng A, Ralph J. Cell wall esterified phenolic dimers: identification and quantification by reverse phase high performance liquid chromatography and diode array detection. Phytochem Anal. 1996;7:305-12.

17. Dai J, Mumper RJ. Plant Phenolics: extraction, analysis and their antioxidant and anticancer properties. Molecules. 2010;15:7313-52.

18. García-Lara S, Bergvinson DJ. Phytochemical and nutraceutical changes during recurrent selection for storage pest resistance in tropical maize. Crop Sci. 2014;54:2423.

19. González-Muñoz A, Quesille-Villalobos AM, Fuentealba C, Shetty K, Gálvez Ranilla L. Potential of Chilean native corn (Zea mays L.) accessions as natural sources of phenolic antioxidants and in vitro bioactivity for hyperglycemia and hypertension management. J Agric Food Chem. 2013;61:10995-1007.

20. Žilić S, Serpen A, Akillioğlu G, Gökmen V, Vančetović J. Phenolic compounds, carotenoids, anthocyanins, and antioxidant capacity of colored maize (Zea mays L.) kernels. J Agric Food Chem. 2012;60:1224-31.

21. Lopez-Martinez LX, Oliart-Ros RM, Valerio-Alfaro G, Lee CH, Parkin KL, Garcia HS. Antioxidant activity, phenolic compounds and anthocyanins content of eighteen strains of Mexican maize. LWT Food Sci Technol. 2009:42:1187-92.

22. Pérez-Jiménez J, Torres JL. Analysis of nonextractable phenolic compounds in foods: the current state of the art. J Agric Food Chem. 2011;59:12713-24. 
23. Verma B, Hucl P, Chibbar RN. Phenolic acid composition and antioxidant capacity of acid and alkali hydrolysed wheat bran fractions. Food Chem. 2009;116:947-54.

24. Classen D, Arnason JT, Serratos JA, Lambert JD, Nozzolillo C, Philogéne BJ. Correlation of phenolic acid content of maize to resistance to Sitophilus zeamais, the maize weevil, in CIMMYT's collections. J Chem Ecol. 1990;16:301-15.

25. Kim K-H, Tsuo R, Yang R, Cui SW. Phenolic acid profiles and antioxidant activities of wheat bran extracts and the effect of hydrolysis conditions. Food Chem. 2006;95:466-73.

26. Mattila P, Kumpulainen J. Determination of free and total phenolic acids in plant-derived foods by HPLC with diode-array detection. J Agric Food Chem. 2002;50:3660-7.

27. Liu B, Li W, Hu L, Zhao J. Mild alkaline hydrolysis is an efficient and lowcost method for improving the free phenolic content and health benefit of pomegranate peel extract. J Food Process Preserv. 2012;37:694-700.

28. Friedman $\mathrm{M}$, Jurgens $\mathrm{H}$. Effect of $\mathrm{pH}$ on stability of plant phenolic compounds. J Agric Food Chem. 2000;48:2101-10.

29. Khoddami A, Wilkes MA, Roberts TH. Techniques for analysis of plant phenolic compounds. Molecules. 2013;18:2328-75.

30. Erez AJ, Ineiro JOS. Effect of solvent, temperature, and solvent-to-solid ratio on the total phenolic content. J Agric Food Chem. 2005;53:2111-7.

31. Cicco N, Lattanzio V. The influence of initial carbonate concentration on the Folin-Ciocalteu micro-method for the determination of phenolics with low concentration in the presence of methanol: a comparative study of real-time monitored reactions. Am J Anal Chem. 2011;2:832-9.

32. Sánchez-Rangel JC, Benavides J, Heredia JB, Cisneros-Zevallos L, JacoboVelázquez DA. The Folin-Ciocalteu assay revisited: improvement of its specificity for total phenolic content determination. Anal Methods. 2013;5:5990.
33. Walker RB. A thorough study of reactivity of various compound classes towards the Folin-Ciocalteu. J Agric Food Chem. 2014;58:8139-44.

34. Andjelkovic M, Van Camp J, Pedra M, Renders K, Socaciu C, Verhé R. Correlations of the phenolic compounds and the phenolic content in some Spanish and French olive oils. J Agric Food Chem. 2008;56:5181-7.

35. OECD. Consensus document on compositional considerations for new varieties of maize (Zea mays): key food and feed nutrients, anti-nutrients and secondary plant metabolites. 2002.

36. Sanahuja G, Farré G, Bassie L, Zhu C, Christou P, Capell T. Ascorbic acid synthesis and metabolism in maize are subject to complex and genotype-dependent feedback regulation during endosperm development. Biotechnol J. 2013;8:1221-30.

37. Arnason JT, Baum B, Gale J, Lambert JDH, Bergvinson D, Philogene BJR, et al. Variation in resistance of Mexican landraces of maize to maize weevil Sitophilus zeamais, in relation to taxonomic and biochemical parameters. Euphytica. 1993;74:227-36.

38. Bily AC, Reid LM, Taylor JH, Johnston D, Malouin C, Burt AJ, et al. Dehydrodimers of ferulic acid in maize grain pericarp and aleurone: resistance factors to Fusarium graminearum. Phytopathology. 2003;93:712-9.

39. García-Lara S, Burt AJ, Arnason JT, Bergvinson DJ. QTL mapping of tropical maize grain components associated with maize weevil resistance. Crop Sci. 2010;50:815.

40. Arnason JT, Conilh B, Philogene BJR, Bergvinson DJ. Mechanisms of resistance in maize grain to the maize weevil and the larger grain borer. In: Mihm JA, editor. Insect resistant maize recent advances and utilization. México: CIMMYT; 1994. p. 91-5.

41. Perkins TJ, Weibe AY, Swain PS. Chance and memory. In: Wall ME, editor. Quantitative biology. Boca Raton: CRC Press; 2013. p. 51-72.

\section{Submit your next manuscript to BioMed Central and we will help you at every step:}

- We accept pre-submission inquiries

- Our selector tool helps you to find the most relevant journal

- We provide round the clock customer support

- Convenient online submission

- Thorough peer review

- Inclusion in PubMed and all major indexing services

- Maximum visibility for your research

Submit your manuscript at www.biomedcentral.com/submit
() Biomed Central 